\title{
Why do some traits show higher fluctuating asymmetry than others? A test of hypotheses with tail feathers of birds
}

\author{
JM Aparicio ${ }^{1}$ and R Bonal ${ }^{2}$ \\ ${ }^{1}$ Instituto de Investigación en Recursos Cinegéticos (IREC), CSIC-UCLM-JCCM, Ronda de Toledo s/n, E-13005 Ciudad Real, Spain; \\ ${ }^{2}$ Departamento de Ciencias Ambientales, Facultad de Ciencias del Medio Ambiente, Universidad de Castilla-La Mancha, E-45071 \\ Toledo, Spain
}

\begin{abstract}
Certain characters are more susceptible to increased fluctuating asymmetry (FA) than others. This trait-specific susceptibility has normally been attributed to different degrees of developmental stability, which could be caused by different modes of selection, functionality, or the stress experienced during the development process. Recently, it has also been suggested that the expression of FA not only depends on developmental stability, but also on the cost of growth of the trait, defined as the amount of structural components necessary to form a unit of length of a given character. In accordance with this argument, a trait with more structural components per unit of length should show lower asymmetry than a simpler one. To test this hypothesis, we examine the structure (number of barbs, barb length, and rachis width)
\end{abstract}

and asymmetry of the longest tail feathers in 26 bird species. Regression analyses using phylogenetically independent contrasts show that FA is negatively correlated with the number of barbs and feather rachis width in males (including species with elongated tails subjected to sexual selection), and with rachis width in females, whose tails supposedly evolve by natural selection. Moreover, the negative correlation between FA and rachis width persisted when taking only the males of non-dimorphic species. These results confirm the hypothesis, suggesting that a trait's susceptibility to express developmental instability by fluctuating asymmetry depends on its structural composition.

Heredity (2002) 89, 139-144. doi:10.1038/sj.hdy.6800118

Keywords: developmental stability; feather structure; ornaments; fluctuating asymmetry

\section{Introduction}

Developmental instability and fluctuating asymmetry (FA) are two concepts frequently used synonymously. Developmental stability (DS) refers to the capability of producing a predetermined phenotype under specific environmental and genetic conditions (Zakharov and Graham, 1992), and is related to the processes that reduce phenotypic variation resulting from small and random disturbances (developmental noise) occurring during the development process at or below the cellular level (Mather, 1953; Waddington, 1957; Zakharov, 1989). Fluctuating asymmetries are small, random deviations from symmetry that occur in the development of bilaterally symmetrical characters (VanValen, 1962), and are commonly used as a measure of developmental stability. Thus, a high level of FA is assumed to reflect reduced developmental stability.

There are certain characters that are more susceptible to increased fluctuating asymmetry than others (eg,

Correspondence: JM Aparicio, Instituto de Investigación en Recursos Cinegéticos (IREC), CSIC-UCLM-JCCM, Ronda de Toledo s/n, E-13005 Ciudad Real, Spain.E-mail: jmaparic@irec.uclm.es

7 September 2001; accepted 28 April 2002
Møller and Swaddle, 1997; Clarke, 1998). Assuming equivalence between developmental stability and fluctuating asymmetry, differences in FA between traits are invariably explained by different levels of developmental stability, which may vary depending on either: (1) trait functionality, (2) mode of selection, and (3) stress associated to the development process.

The degree of fluctuating asymmetry of a trait could depend on its functional importance, because a stabilised development should be more strongly selected in the characters performing critical functions for an organism, in which FA might be detrimental, than in those characters functionally less important (Gummer and Brigham, 1913; Palmer and Strobeck, 1986; Balmford et al, 1993).

Developmental stability could also be reduced by a process of directional selection, since this selective process presumably acts against genetic modifiers that control the expression of the genotype, hence increasing the level of developmental instability (eg, Clarke, 1997). Thus, in the case of secondary sexual traits, under directional selection, it is believed that these show higher asymmetry values than ordinary morphological traits (eg, Møller and Höglund, 1991; Møller and Eriksson, 1994; Dufour and Weatherhead, 1996; Hunt and Simmons, 1998; Cuervo and Møller, 1999) due to the effects of genomic stress on developmental stability that are imposed by their distinct mode of selection (Møller, 
1993a; Møller and Pomiankowski, 1993). However, in other comparative studies no differences have been found in FA between sexual and ordinary morphological traits (Balmford et al, 1993; Tomkins and Simmons, 1995; Leung and Forbes, 1996; Bjorksten, et al, 2000b).

In addition, the levels of developmental instability could increase with the degree of either environmental or genetic stress (reviews in Palmer and Strobeck, 1986; Leary and Allendorf, 1989; Parsons, 1990, 1992; Møller and Swaddle, 1997). Thus, because some characters are exaggeratedly developed, such as ornaments, are presumably very costly to produce, it has been hypothesised that this exaggeration would itself cause stress and lead to increased FA values (Møller and Pomiankowski, 1993), or to a greater sensitivity in the effect of environmental stress on fluctuating asymmetry (Møller, 1990, 1993b). Nevertheless, some experimental studies have found no evidence of an increase in the FA of secondary sexual traits growing under stressful conditions (eg, Hunt and Simmons, 1997; David et al, 1998; Bjorksten et al, 2000a).

We do not question the effects of developmental stability on fluctuating asymmetry or the causes of a lowered developmental stability, but we think that another factor may mediate the link between developmental stability and fluctuating asymmetry (Aparicio, 1998, 2001). An indirect causal relationship between DS and FA could be even more likely than a direct one, taking into account that the processes related to DS are probably operating at the microscopic level, whereas fluctuating asymmetry is the macroscopic outcome of unrepaired microscopic errors. So, the translation of microscopic errors to the macroscopic asymmetries found in the character could depend on a third factor, such as the amount of structural components needed to form a unit of length of a given character. Thus, an asymmetrical allocation of resources to both sides of a trait as a consequence of developmental instability would produce FA in the trait, but the same magnitude in asymmetrical allocation will result in a higher asymmetry in those characters that need less structural components for a unit of length to be formed (Figure 1). According to this model, differences in asym-

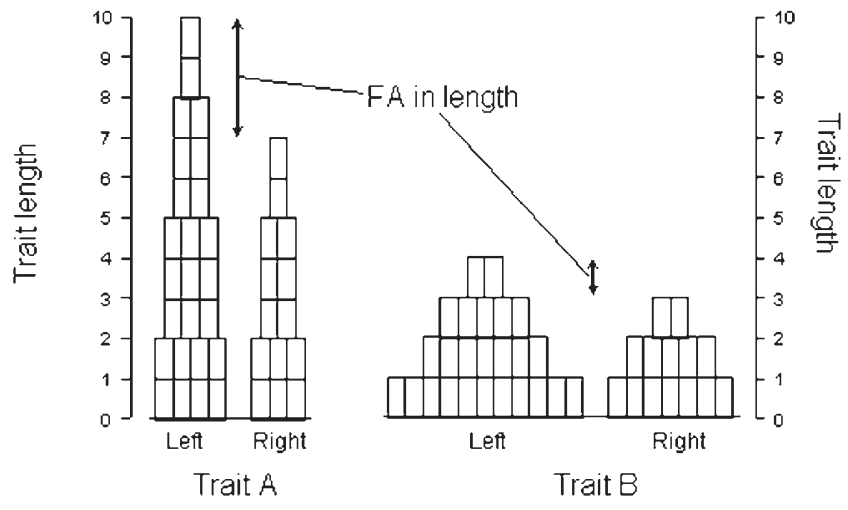

Figure 1 Effect of structural composition on fluctuating asymmetry (FA). Both trait A and trait B vary their structure with the length to maintain their mechanical properties following a certain rule. At equal length, Trait A has less components per unit length than Trait 2. Equal asymmetry in number of components devoted to develop left sides ( 25 units) and right sides (14 units) cause different degree of asymmetry in trait length, being FA lower as more elements compose a unit of trait length. metry between two traits do not necessarily only indicate differences in developmental stability, mode of selection or degree of stress, but may also arise from differences in the structural composition of the character. Thus, traits with less structural components per unit length should be more likely to show FA than those with a denser structure.

To test the structural hypothesis, we examined the structure of tail feathers, measured as rachis width, and the number and length of barbs in 26 species. All components of a feather consist basically of keratin and have a common origin from a feather folicle (Price et al, 1991; Prum, 1999), and their amino acid profiles are remarkably similar among all their components and among species representing different orders and varied lifestyles (Harrap and Woods, 1967; King and Murphy, 1987; Murphy et al, 1990; Vincent, 1990). Therefore, if this hypothesis were correct, we would expect a negative correlation between the size and number of these structural components and the degree of fluctuating asymmetry.

\section{Methods}

We examined the left and the right longest tail feathers on museum skins of 26 bird species. To choose these species, we established four categories defined by the dimorphism in tail length (dimorphic and nondimorphic) and within these by the functionality of the tail (functional and non-functional tails). We considered a species as dimorphic if the tail length of the male is 5\% longer than that of the female (a value of reference normally considered in the literature a classifying a species as sexually dimorphic, eg, Cuervo and Møller 1999). The tails were defined as functional if these were used for aerial foraging, as body stabilizers in diving species, or to provide support on the tree trunk. Within these four categories, the species were chosen randomly among those available at three Museum collections consulted. Besides this, and in order to discern between the structural and the functional-aerodynamic hypotheses, we also included three bird species whose tails are used to prop them up against tree trunks rather than for flight. The exclusion/inclusion of these species did not affect the other results. For each species we took at least five individuals of each sex in full breeding plumage, with the proviso that they did not show any sign of moulting or damage to their tails. We recorded feather length, number of barbs per $5 \mathrm{~mm}$, barb length, and rachis width in the middle of the feather. Measurements of rachis were made with digital calliper $\pm 0.01 \mathrm{~mm}$, and further barbs were measured under a binocular microscope with $\times 5$ 10 magnification. The length was measured from the insertion of the calamus into the skin to the tip of the rachis. These measures were taken four times on each feather, interchanging the right and the left sides. A mixed-model ANOVA, as suggested by Swaddle et al (1994) was employed to test the repeatability of individual measures of asymmetry. Asymmetry estimates were significantly repeatable for all species as the lowest value was: $F_{8,48}=7.28 ; P<0.0001$.

We used a dimorphic index for tail feathers which is given by difference of $\log _{10}$ male minus $\log _{10}$ female feather lengths. Because fluctuating asymmetry is expected to increase with feather length, it is necessary 
to correct by size when comparing FA between different species (Palmer and Strobeck, 1986; Leung, 1998; Windig and Nylin, 2000). Males of dimorphic species have enlarged their tails at the same time that they have increased their asymmetries. These asymmetry values may partly be due to the effect of their long tails per se, or to the effect of lower developmental stability caused by directional selection (see above). To remove the effect of long tails without deleting possible effects of directional selection, we examined the relationship between log-transformed values of asymmetry and feather length in males of non-dimorphic species (dimorphic index $<5 \%$ ) and females, whose FA is presumably due only to the effect of length. An ANCOVA showed that there was no difference between sexes in the regression line (constant: $F_{1,34}=0.01, P=0.92$; slope: $F_{1,34}=0.08, P=0.78$ ). The regression line resulting from these analyses $\left(\mathrm{FA}=0.073\right.$ feather length $\left.{ }^{0.48}\right)$ explained a significant proportion of variance $\left(r^{2}=21 \%, P=0.004\right)$, and was used to standardise FA values of dimorphic and non-dimorphic species. Relative fluctuating asymmetry was given as the difference of observed minus expected fluctuating asymmetry.

Data were analysed with the phylogeny-based statistical method of independent contrasts (Felsenstein, 1985). Independent contrasts were generated in the PDTREE program (Garland et al, 1992, 1993, 1999) and standardised before being analysed. All regressions on contrasts were forced through the origin (Garland et al, 1992).

We used the phylogeny constructed by Sibley and Ahlquist (1990) and took supplementary information from Bleiweiss et al (1997) for hummingbirds, and Swierczewski and Raikow (1981) for piciformes. Phylogenetic contrast given in this paper were obtained by taking branch lengths as the delta $\mathrm{H}_{50} \mathrm{~T}$ values (Figure 2). Nevertheless, all analyses were repeated considering all branches of length equal to a unit, and the results did not change qualitatively.

\section{Results}

Comparisons of raw data showed that males showed greater relative fluctuating asymmetry than females (Wilcoxon paired test: $z=2.27, n=26, P=0.02$ ). Moreover, there was a positive correlation between the contrasts for sexual dimorphism in tail length and males' relative asymmetry of tail feathers $(r=0.59, n=25$, $P=0.0015$; Figure 3). In agreement with previous studies, these results could suggest that higher FA values of ornamental tails are due to the effects of directional selection, if males belonging to more dimorphic species are also those undergoing stronger directional selection on the lengths of their tails.

We also found that males' ornamental tails had a simpler structure than non-ornamental ones, since the degree of dimorphism in tail feathers was negatively and significantly associated with both, rachis width $(r=-0.43$, $n=25, P=0.028)$ and number of barbs per unit of feather length $(r=-0.59, n=25, P=0.001)$, but not with barb length $(r=-0.04, n=25, P=0.85)$. According with Aparicio's (2001) model, these differences in structural composition could also account for differences in asymmetry between the two kinds of feathers.

To distinguish between the effects of structural compo-

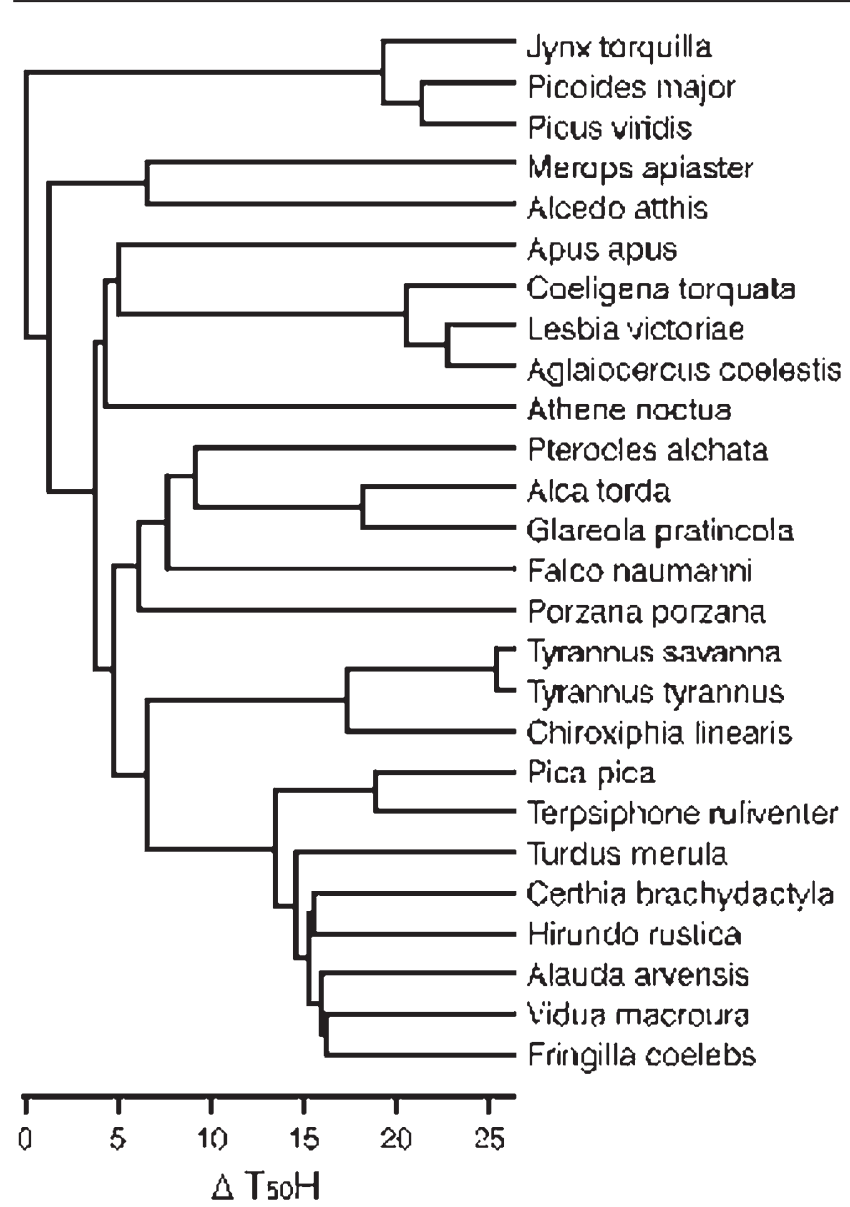

Figure 2 Hypothesised phylogenetic relationships among the 26 species of birds.

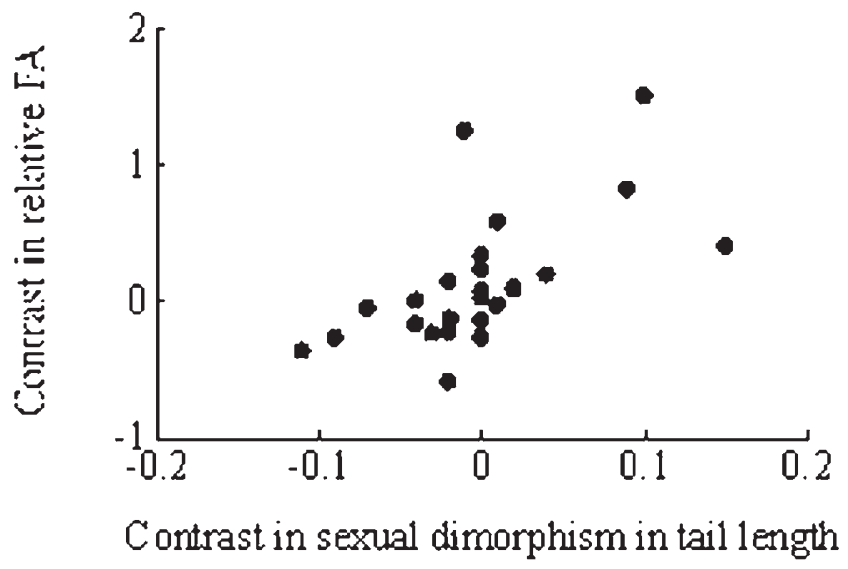

Figure 3 Relationship between sexual dimorphism in tail length and the level of relative fluctuating asymmetry (FA) in tail feathers.

sition and those resulting from the mode of selection, we performed multiple regression analyses including phylogenetic contrasts in relative FA as the dependent variable and phylogenetic contrast in number of barbs, barb length, rachis width, and degree of dimorphism in tail feather length as independent variables. For males, the analysis showed a significant correlation between relative asymmetry and both rachis width $(\beta=-0.52, t=3.55$, 
$P=0.002 ;$ Figure $4 \mathrm{a})$, and number of barbs $(\beta=-0.58$, $t=3.31, P=0.003$; Figure $4 \mathrm{~b})$, but neither barb length ( $\beta=0.03, t=0.21, P=0.83$, power test: 0.05$)$ nor degree of dimorphism were significant $(\beta=0.07, t=0.42, P=0.68$, power test: 0.06$)$. The male database was split in two subgroups: one of males of species dimorphic for tail length $(\geq 5 \%)$, and other of males of non-dimorphic species $(<5 \%)$. In dimorphic species, the relative fluctuating asymmetry of males was still associated with rachis width $(\beta=-0.66, t=3.59, P=0.005$; Figure $4 \mathrm{c})$, and number of barbs $(\beta=-0.81, t=4.22, P=0.002$; Figure $4 \mathrm{~d})$, but not with barb length $(\beta=-0.32, t=1.12, P=0.3$; power test: 0.2$)$ or degree of dimorphism $(\beta=-0.42, t=1.9$, $P=0.09$; power test: 0.4$)$. Furthermore, feather structure had also a negative effect on relative fluctuating asymmetry of both females and males of non-dimorphic species, whose tails are not apparently subject to sexual selection. In females, rachis width explained a high percentage of variance in females' relative fluctuating asymmetry significant $(\beta=-0.59, t=3.05, P=0.68$, Figure $4 \mathrm{e})$, whilst barb length $(\beta=0.17, t=0.78, P=0.44$, power test: $0.13)$, number of barbs $(\beta=-0.27, t=1.30, P=0.21$, power test: 0.19$)$, and degree of dimorphism $(\beta=0.16, t=0.77$, $P=0.45$, power test: 0.15$)$ were not significantly associated with relative FA. In non-dimorphic males there was a significant negative correlation between relative FA and rachis width $(r=0.74, n=12, P=0.006$; Figure $4 f)$, but with no other variable.

Eight of the studied species normally forage in flight (Merops apiaster, Apus apus, Lesbia victoriae, Aglaiocercus coelestis, Coeligena torquata, Glareola pratincola, Falco naum-
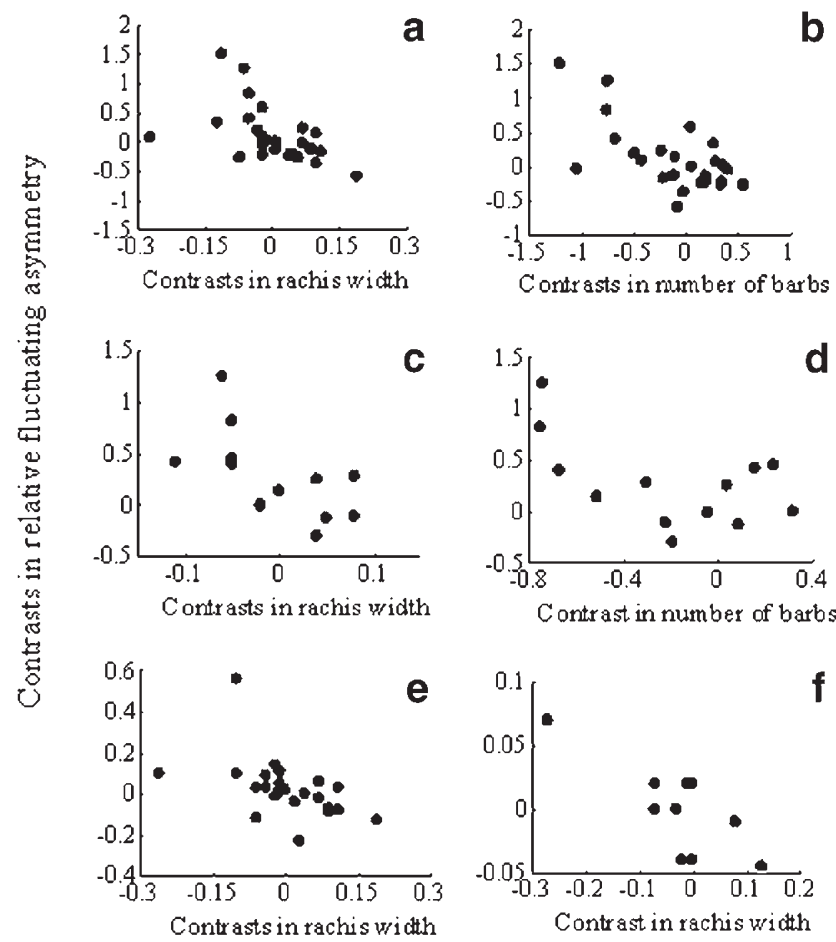

Figure 4 Relationships between phylogenetic contrast in relative fluctuating asymmetry in tail feathers and contrast in feather components measured as rachis width and number of barbs. $(\mathbf{a}, \mathbf{b})$ males of all studied species. (c,d) males of species with tail dimorphism greater than 5\%. (e) female of all studied species (f) nondimorphic males.
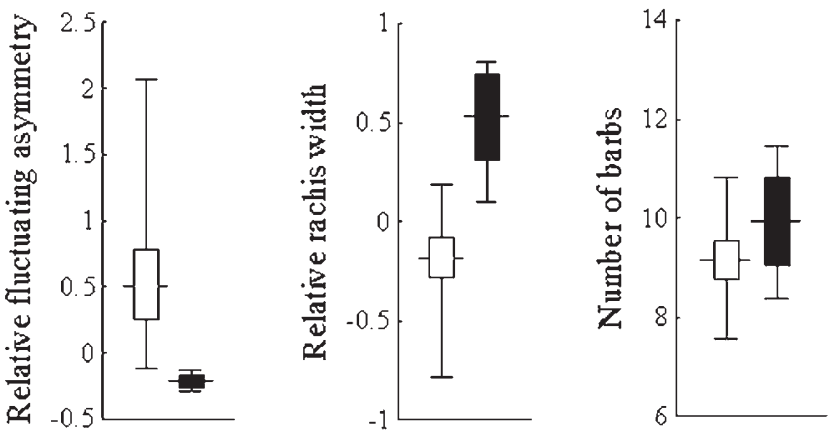

Figure 5 Comparisons of relative fluctuating asymmetry, rachis width and number of barbs in tail feather of aerial foragers (open bars include the following species: Merops apiaster, Apus apus, Lesbia victoriae, Aglaiocercus coelestis, Coeligena torquata, Glareola pratincola, Falco naumanni, and Hirundo rustica) versus species using their tails to sustain their bodies against the trunks of trees (black bars include the species: Picoide major, Picus viridis, and Certhia brachydactyla).

anni, and Hirundo rustica). All of these showed greater mean relative FA in tail feathers than did three species, two woodpeckers (Picoides major, and Picus viridis), and the treecreeper, Certhia brachydactyla, whose tails serve to prop the bird up against the trunks of trees (MannWhitney $U$-test: $z=2.45, P=0.01)$. These results could be accounted for by differences in structural composition, as rachis, width relative to feather length was thinner for aerial foragers than for treecreepers and woodpeckers $(U-$ test: $z=2.04, P=0.04$ ), whilst there was no significant difference in the number of barbs (U-test: $z=0.92, P=0.4$; Figure 5).

\section{Discussion}

Variations in the level of fluctuating asymmetry between traits have generally been attributed to differences in their degree of developmental stability, which may change depending on either: trait functionality, mode of selection, or the degree of stress experienced during character production. However, the relationship between stress and FA is not always clear and often subject to controversy (review in Bjorksten et al, 2000b). Recently, Aparicio (2001) has proposed a model on the origin of fluctuating asymmetry, which predicts that FA levels could also depend on the amount of structural components necessary to form a unit of length of a trait without considering ecological costs. Thus, for a given degree of developmental stability, there would be a negative association between FA and the structural density of a trait. Our results seem to support the prediction of the model since an examination of the feather structure and asymmetry in 26 species showed that relative (corrected for feather length) fluctuating asymmetries were negatively correlated with the number of barbs and the feather rachis width.

As in some previous studies, we found that males with ornamental tails had higher asymmetry values in their tail feathers than females of the same species, and when compared with males of different species without ornamental tails (eg, Møller and Höglund, 1991; Møller and Eriksson, 1994; Dufour and Weatherhead 1996; Hunt and Simmons, 1998; but see for example Balmford et al, 1993; Tomkins and Simmons, 1995; Leung and Forbes, 1996; 
Bjorksten et al, 2000b, for contrary results). Moreover, if males belonging to more dimorphic species are also supporting a stronger directional selection on the lengths of their tails, the positive correlation between degree of dimorphism in tail feathers and their asymmetry could be supporting the hypothesis proposed by Møller and Pomiankowski (1993), on the effects of sexual selection on FA values. However, when trait structure was taken into account, the degree of dimorphism was not significantly related to the level of fluctuating asymmetry. Therefore, our results indicate that ornamental tail feathers are more asymmetrical than non-ornamental feathers, since the first ones have evolved a shortening in the number and size of their structural components per unit of length. In fact, the degree of dimorphism is negatively correlated with the number of barbs and rachis width. Similar results were also reported by Møller and Hedenström (1999), who found that ornamental feathers have a narrower vane than non-ornamental ones. Hence, ornamental tails seem to include less structural components per unit of length than non-ornamental ones, and this probably favours a greater susceptibility to express FA.

In support of the structural hypothesis, the analyses dealing with females, whose tails are supposedly not subject to sexual selection, gave similar results to those for the males. Moreover, including only males of non-dimorphic species, rachis width was also negatively associated with the degree of fluctuating asymmetry. However, there was no significant association between number of barbs and FA, presumably because there was little variability in the value of this parameter between non-dimorphic species.

Differences in tail fluctuating asymmetry among species have also been attributed to the functionality of the trait. For example, Balmford et al (1993) suggested that degree of fluctuating asymmetry of a trait could depend on its functional importance for flight, as FA could cause important aerodynamic costs. The structural hypothesis is not contrary to the functional hypothesis, since besides a selection for symmetry - functional traits may have a more elaborated structure (and so lower FA) than non-functional traits. Our results seem to support the hypothesis suggesting that the relationship between the level of FA and the functionality of a character could result from an association between functionality and structural composition in addition to an association between functionality and selection against asymmetry. In fact, tail asymmetry is apparently more costly for aerial foragers than it is for treecreepers and woodpeckers (see Balmford et al, 1993), but these last two groups show lower tail asymmetry values than do aerial foragers. These differences may be explained by the different structure of their feathers, since treecreepers, and especially woodpeckers, due to the need of physical resistance of their tail feathers, have developed these to be more robust and structurally denser than those of aerial foragers.

In summary, the amount of structural components necessary to produce a unit of length of a trait has a great influence in the expression of developmental instability in asymmetry, and thus, more elaborate characters should normally show lower asymmetry values than those less elaborate. This dependence of FA on structural composition should be taken into account in future stud-

ies on FA, and it could give insight into the current controversy about the usefulness of FA as an indicator of stress.

\section{Acknowledgements}

We thank Museo Nacional de Ciencias Naturales, British Museum of Natural History, Estación Biológica de Doñana, for loaning some specimens to carried out this study. $\mathrm{RB}$ is grateful to José $\mathrm{P}$ Veiga for allowing his stay at the Museo Nacional de Ciencias Naturales. This study was partially supported by the project PB97-1249.

\section{References}

Aparicio JM (1998). Patterns of fluctuating asymmetry in developing primary feathers: a test of the compensational growth hypothesis. Proc $R$ Soc Lond B 265: 2353-2357.

Aparicio JM (2001). Patterns of growth and fluctuating asymmetry: the effects of asymmetrical investment in traits with determinate growth. Behav Ecol Sociobiol 49: 273-272.

Balmford A, Jones IL, Thomas ALR (1993). On avian asymmetry: evidence of natural selection for symmetrical tails and wings in birds. Proc $R$ Soc Lond B 252: 245-251.

Bjorksten TA, David P, Pomiankowski A, Fowler K (2000a). Fluctuating asymmetry of sexual and nonsexual traits in stalkeyed flies: a poor indicator of developmental stress and genetic quality. J Evol Biol 13: 89-97.

Bjorksten TA, Fowler K, Pomiankowski A (2000b). What does sexual trait FA tell us about stress? TREE 15: 163-166.

Bleiweiss R, Kirsch JAW, Matheus JC (1997). DNA hybridization evidence for the principal lineages of hummingbirds. Mol Biol Evol 14: 325-343.

Clarke GM (1997). The genetic and molecular basis of developmental stability: the Lucilia story. TREE 12: 89-91.

Clarke GM (1998). The genetic and molecular basis of developmental stability. V. Inter- and intra-individual character variation. Heredity 80: 562-567.

Cuervo JJ, Møller AP (1999). Phenotypic variation and fluctuating asymmetry in sexually dimorphic feather ornaments in relation to sex and mating system. Biol J Linn Soc 68: 505-529.

David P, Hingle A, Greig D, Rutherford A, Pomiankowski A, Fowler K (1998). Male sexual ornament size but not asymmetry reflects condition in stalk-eyed flies. Proc $R$ Soc Lond $B$ 265: 2211-2216.

Dufour K, Weatherhead PJ (1996). Estimation of organism-wide asymmetry in red-winged blackbirds and its relation to studies of mate selection. Proc R Soc Lond B 263: 769-775.

Felsenstein J (1985). Phylogenies and the comparative method. Am Nat 125: 1-15.

Garland Jr T, Harvey PH, Ives AR (1992). Procedures for the analysis of comparative data using phylogenetically independent contrasts. Syst Biol 41: 18-32

Garland Jr T, Dickerman AW, Janis CM, Jones JA (1993). Phylogenetic analysis of covariance by computer simulation. Syst Biol 42: 265-292.

Garland Jr T, Midford PE, Ives AE (1999). An introduction to phylogenetically based statistical methods, with a new method for confidence intervals on ancestral values. Am Zool 39: 374-388.

Gummer DL, Brigham RM (1995). Does fluctuating asymmetry reflect the importance of trait in little brown bats (Myotis lucifugus)? Can J Zool 73: 990-992.

Harrap BS, Woods EF (1967). Species differences in the proteins of feathers. Comp Biochem Physiol 20: 449-460.

Hunt J, Simmons LW (1997). Patterns of fluctuating asymmetry in beetle horns: an experimental examination of the honest signalling hypothesis. Behav Ecol Sociobiol 41: 109-114.

Hunt J, Simmons LW (1998). Patterns of fluctuating asymmetry in beetle horns: no evidence for reliable signaling. Behav Ecol 9: $465-470$. 
King JR, Murphy ME (1987). Amino acid composition of the calamus rachis and barbs of White-crowned Sparrow feathers. Condor 89: 436-439.

Leary RF, Allendorf FW (1989). Fluctuating asymmetry as an indicator of stress: implications for conservation biology. TREE 4: 214-217.

Leung B (1998). Correcting for allometry in studies of fluctuating asymmetry and quality within samples. Proc $R$ Soc Lond B $\mathbf{2 6 5}$ : 1623-1629.

Leung B, Forbes MR (1996). Fluctuating asymmetry in relation to stress and fitness: effects of trait type as revealed by metaanalysis. Ecoscience 3: 400-413.

Mather K (1953). Genetical control of stability in development. Heredity 7: 297-336.

Møller AP (1990). Fluctuating asymmetry in male sexual ornaments may reliably reveal male quality. Anim Behav 40: 1185-1187.

Møller AP (1993a). Developmental stability, sexual selection and speciation. J Evol Biol 6: 493-509.

Møller AP (1993b). Morphology and sexual selection in the barn swallow Hirundo rustica in Chernobyl, Ukraine. Proc $R$ Soc Lond B 252: 51-57.

Møller AP, Eriksson M (1994). Patterns of fluctuating asymmetry in flowers: implications of honest signalling for pollinators. $J$ Evol Biol 7: 97-113.

Møller AP, Hendenström A (1999). Comparative evidence for costs of secondary sexual characters: adaptive vane emargination of ornamented feathers in birds. J Evol Biol 12: 296-305.

Møller AP, Höglund J (1991). Patterns of fluctuating asymmetry in avian feather ornaments: implications for models of sexual selection. Proc R Soc Lond B 245: 1-5.

Møller AP, Pomiankowski A (1993). Fluctuating asymmetry and sexual selection. Genetica 89: 267-279.

Møller AP, Swaddle JP (1997). Asymmetry, Developmental Stability and Evolution. Oxford University Press: Oxford.
Murphy ME, King JR, Taruscio TG, Geupel GR (1990). Amino acid composition of feather barbs and rachises in three species of pygoscelid penguins: nutritional implications. Condor 92: 913-921.

Palmer AR, Strobeck C (1986). Fluctuating asymmetry: measurements, analysis, patterns. Ann Rev Ecol Syst 17: 391-421.

Parsons PA (1990). Fluctuating asymmetry: an epigenetic measure of stress. Biol Rev 65: 131-145.

Parsons PA (1992). Fluctuating asymmetry: a biological monitor of environmental and genomic stress. Heredity 68: 361-364.

Price T, Chi E, Pavelka M, Hack M (1991). Population and developmental variation in the feather tip. Evolution 45: 518-533.

Prum RO (1999). Development and evolutionary origin of feathers. J Exp Zool 285: 291-306.

Sibley CG, Ahlquist JE (1990). Phylogeny and Classification of Birds. A Study in Molecular Evolution. Yale University Press.

Swaddle JP, Witter MS, Cuthill IC (1994). The analysis of fluctuating asymmetry. Anim Behav 48: 986-989.

Swierczewski EC, Raikow RJ (1981). Hind limb morphology, phylogeny, and classification of the Piciformes. Auk 98: 466480 .

Tomkins JL, Simmons LW (1995). Patterns of fluctuating asymmetry in earwig forceps: no evidence for reliable signalling. Proc R Soc Lond B 259: 89-96.

Van Valen L (1962). A study of fluctuating asymmetry. Evolution 16: $125-142$.

Vincent J (1990). Structural Biomaterials. Princeton University Press: Princeton, NJ.

Waddington CH (1957). The Strategy of the Genes. George Allen Unwin: London.

Windig JJ, Nylin S (2000). How to compare fluctuating asymmetry of different traits. J Evol Biol 13: 29-37

Zakharov VM (1989). Future prospects for population phenogenetics. Soviet Sci Rev, Sect F, Physiol Gen Biol 4: 1-79.

Zakharov VM, Graham JH (1992). Introduction. In Developmental stability in natural populations. Acta Zool Fenn 191: 4-5 